\title{
Lutilizzo degli strumenti di lavoro nell'ambulatorio dei pediatri di famiglia in provincia di Bergamo: un impiego eterogeneo?
}

\author{
Romeo Carrozzo, Irene Bonicelli, Marzia Bacchini, Monica Altobelli, \\ Chiara Caldiani, Nicoletta Pellegrini, Alberto Vitali, Danila Mariani \\ Pediatri di libera scelta, ATS Bergamo
}

\begin{abstract}
Obiettivi: abbiamo studiato l'omogeneità nell'utilizzo di alcuni strumenti di lavoro in un gruppo di pediatri di libera scelta operanti nella Provincia di Bergamo.

Metodi: attraverso la compilazione online di un questionario, abbiamo esaminato in maniera retrospettiva i dati raccolti da 85 pediatri relativi al periodo 24 febbraio- 3 maggio 2019.

Risultati: l'analisi dei dati ha dimostrato un maggiore utilizzo del self-help per i pediatri che si avvalgono del lavoro di personale infermieristico $(p=0,03)$, e un più basso numero di visite ambulatoriali per i pediatri che non si avvalgono di personale nella gestione dell'ambulatorio $(p=0,06)$. Abbiamo registrato un grado di eterogeneità sostanzialmente doppio nell'uso degli strumenti di self-help rispetto a quello riscontrato per altri strumenti di lavoro (coefficiente di variazione 0,66 vs $0,27-0,37$ ).

Conclusioni: questi dati suggeriscono l'opportunità di formulare indicazioni condivise riguardanti l'uso degli strumenti di lavoro per gli ambulatori pediatrici del territorio.
\end{abstract}

\begin{abstract}
Objectives: we investigated on the degree of homogeneity in the employment of some working tools among primary care paediatricians working in the province of Bergamo.

Methods: through the compilation of an online questionnaire, we retrospectively evaluated data recorded from February 24 to May 3 2019, by 85 paediatricians.

Results: the data analysis showed a larger employment of point of care testing for

paediatricians relying on nurses activity $(p=0.03)$, and a lower number of outpatient visits for paediatricians without office staff $(p=0.06)$. A rough doubling in heterogeneity was assessed in the employment of point of care testing when compared to the other working tools (coefficient of variation 0.66 vs. $0.27-0.37$ ).

Conclusions: these data address to the worthiness of shared procedures referring to the employment of working tools in paediatric primary care.
\end{abstract}

\section{Introduzione}

Luso di linee guida, protocolli e percorsi diagnostico terapeutici assistenziali (PDTA) costituiscono un elemento centrale nella pratica sanitaria moderna. Benché la decisione ultima in termini diagnostico-terapeutici non possa eludere il ricorso all'ars medica maturata dal singolo sanitario, le società scientifiche producono uno sforzo costante per proporre, nei principali scenari clinici, un approccio per quanto possibile omogeneo. Inoltre, le indicazioni generali provenienti da analisi e confronti condivisi hanno ormai raggiunto valenza giuridica [1]. La figura del pediatra di libera scelta (PLS) italiano costituisce un unicum nel panorama sanitario europeo [2]. I termini previsti dall'accordo collettivo nazionale (ACN) con i PLS permettono, pur nell'ambito dei vincoli contrattuali, una notevole duttilità nelle modalità di offerta delle prestazioni sanitarie, privilegiando il rapporto fiduciario quale valore fondante della diade medico-paziente. L'introduzione di forme di pediatria in associazione, oltre ad ampliare l'offerta dei servizi sul territorio, ha tuttavia sottolineato la necessità per i PLS aderenti di "condividere e implementare linee guida diagnostico terapeutiche per le patologie a più alta prevalenza e attuare momenti di verifica periodica" [3].

In relazione all'uso di strumenti di lavoro del PLS, quali i bilanci di salute (BdS), le prestazioni di particolare impegno professionale (PPIP) e la prescrizione dei farmaci, molti studi hanno esaminato le modalità di utilizzo globale di gruppi di pediatri, confrontandone l'aderenza a linee guida e a modalità condivise di impiego [4-7].

Scopo del presente studio, condotto nella Provincia di Bergamo, è stata un'indagine sull'uso di questi stessi strumenti di lavo- ro nell'ambulatorio del PLS, raffrontando i risultati ottenuti per i singoli pediatri in relazione alla presenza di collaboratori (personale di segreteria e/o personale infermieristico), e paragonando i singoli strumenti di lavoro per grado di omogeneità di impiego da parte dei PLS.

\section{Materiali e metodi}

I dettagli relativi all'invio e alla raccolta dei questionari sono stati descritti in altra pubblicazione [8]. In breve, a tutti i Pls della Provincia di Bergamo è stato richiesto di compilare via web un questionario di 26 domande per valutare l'impatto che il periodo di lockdown dovuto alla pandemia da SARS-CoV-2 aveva avuto sulla loro attività ambulatoriale. Per un'analisi comparativa, è stato richiesto di raccogliere retrospettivamente i dati relativi al periodo 24 febbraio-3 maggio per gli anni 2019 e 2020.

Per le finalità del presente studio, sono stati valutati i dati relativi al periodo 24 febbraio-3 maggio 2019 per 5 strumenti di lavoro del pediatra di famiglia: [a] visite ambulatoriali; [b] BdS; [c] PPIP (limitate a test rapido per SBEGA, stick urine, PCR): [d] numero di confezioni prescritte per farmaci di fascia A; [e] numero di confezioni prescritte per antibiotici di fascia $A$. I dati sono stati estratti da software gestionali per gli ambulatori (Junior Bit, Camilla, Infantia, MDB2000). Sono stati utilizzati cinque indicatori: (i) visite/assistibili; (ii) BdS/assistibili; (iii) PPIP/visite; (iv) farmaci fascia A/ visite; (v) antibiotici fascia $\mathrm{A} /$ farmaci fascia A, i cui valori sono stati calcolati per ciascun pediatra.

L'analisi dei dati è stata effettuata mediante il calcolo degli indici statistici descrittivi (medie, indici di variabilità); i confronti tra le medie sono stati eseguiti mediante t-test (significatività statistica con $\alpha=0,05)$. Il valore del coefficiente di variazione (DS/media) è stato assunto come indice di omogeneità per le osservazioni relative ai 5 indicatori individuati. 
Risultati

Sono stati invitati 127 PLS della Provincia di Bergamo alla compilazione del questionario online. Sono state ottenute risposte da 88 pediatri (69\%). Per il presente studio abbiamo utilizzato le risposte fornite da 85 pediatri ( 85.520 assistibili), i cui dati risultavano correttamente compilati per le variabili oggetto di questo lavoro.

I risultati dello studio sono riportati nelle

Tabelle e nelle Figure.

Commento ai risultati

Partendo da un'analisi retrospettiva sull'attività dei PLS della Provincia di
Bergamo durante la fase di lockdown del 2020, abbiamo utilizzato i dati relativi a un periodo di 47 giorni lavorativi nel periodo 24 febbraio-3 maggio 2019 riferiti a 85 PLS, per valutare l'impatto della presenza del personale di segreteria e/o infermieristico sull'impiego di alcuni strumenti di lavoro quotidiano, quali le visite ambulatoriali, l'utilizzo delle PPIP, l'esecuzione dei BdS.

L'analisi dell'indicatore relativo ai BdS (Tabella 2) non ha evidenziato differenze significative fra i pediatri che si avvalgono e quelli che non si avvalgono di personale nella gestione dell'ambulatorio. Benché sia verosimile ipotizzare un diretto coinvolgimento del personale di segreteria e di quello infermieristico nella programmazione e nella erogazione della prestazione, i BdS, introdotti nella contrattualistica nazionale in via sperimentale sin dal 1996, sono ormai uno strumento di lavoro largamente impiegato e gradito alle famiglie [9], per il quale la nostra analisi suggerisce un impiego sostanzialmente uniforme nel campione di pediatri esaminato.

L'indicatore relativo alle visite ambulatoriali ha dimostrato un maggiore impiego di questo strumento per i pediatri che si avvalgono di personale di segreteria/infermieri-

\section{TABELLA 1 Dati cumulativi relativi ai pediatri partecipanti allo studio, alla presenza di personale e agli strumenti} di lavoro analizzati per il periodo 24 febbraio-3 maggio 2019

\begin{tabular}{|l|c|c|c|}
\hline & $\begin{array}{c}\text { Pediatri senza } \\
\text { personale di studio }\end{array}$ & $\begin{array}{c}\text { Pediatri con personale di } \\
\text { studio }\end{array}$ & 57 \\
\hline N. pediatri partecipanti & 28 & $62.078(1089)$ & 85 \\
\hline N. assistibili (media) & $23.442(837)$ & $55(38-65)$ & $16 / 41$ \\
\hline Mediana età pediatra (range) & $48(41-64)$ & 39.744 & $18 / 67$ \\
\hline Genere (M/F) & $2 / 26$ & 6292 & 52.556 \\
\hline Visite ambulatoriali & 12.812 & 3691 & 8740 \\
\hline Bilanci di salute & 2448 & 24.998 & 4955 \\
\hline PPIP & 1264 & 10.902 & 35.029 \\
\hline Confezioni di farmaci in fascia A prescritti & 10.031 & 4389 & 15.291 \\
\hline Confezioni di antibiotici in fascia A prescritti & & & 24 \\
\hline
\end{tabular}

TABELLA 2 Medie e deviazioni standard per indicatore (x100) in relazione alla presenza di personale

\begin{tabular}{|c|c|c|c|c|c|}
\hline Indicatore & $\begin{array}{c}\text { Pediatri senza } \\
\text { personale }\{28\}^{*} \\
{[\mathrm{~A}]}\end{array}$ & $\begin{array}{c}\text { Pediatri con solo } \\
\text { personale di } \\
\text { segreteria }\{35\}^{*} \\
{[B]}\end{array}$ & $\begin{array}{c}\text { Pediatri con } \\
\text { personale di } \\
\text { segreteria ed } \\
\text { infermieristico } \\
\{20\}^{*} \\
{[\mathrm{C}]}\end{array}$ & $\begin{array}{c}\text { Pediatri con solo } \\
\text { personale } \\
\text { infermieristico } \\
\{2\}^{*} \\
{[D]}\end{array}$ & $\mathrm{p}$ value \\
\hline BdS/assistibili (SD) & $104(44)$ & $102(25)$ & $100(23)$ & $80-94^{* *}$ & $\begin{array}{c}>0.5 \\
{[A] \text { vs. }[B] ;[A] \text { vs. }[C] ;} \\
{[B] \text { vs. }[C]}\end{array}$ \\
\hline Visite/assistibili (SD) & $563(166)$ & $639(176)$ & $636(164)$ & $484-685^{* *}$ & $\begin{array}{c}0.06 \\
{[A] \text { vs. }[B, C, D]}\end{array}$ \\
\hline PPIP/visite (SD) & $107(78)$ & $77(49)$ & $127(65)$ & $155-63^{* *}$ & $\begin{array}{c}0.03 \\
{[A, B] \text { vs. }[C, D]}\end{array}$ \\
\hline
\end{tabular}

\section{TABELLA 3. MEDIE, DEVIAZIONI STANDARD E COEFFICIENTE DI VARIAZIONE PER INDICATORE}

\begin{tabular}{|c|c|c|c|}
\hline Indicatore & Media & SD & Coefficiente di variazione \\
\hline Visite/assistibili* & 0,612 & 0,170 & 0,27 \\
\hline BdS/assistibili* & 0,102 & 0,032 & 0,31 \\
\hline PPIP/visite* & 0,099 & 0,066 & 0,66 \\
\hline Farmaci fascia A/visite ${ }^{* *}$ & 0,721 & 0,270 & 0,37 \\
\hline Antibiotici/Farmaci fascia $A^{* *}$ & 0,446 & 0,134 & 0,30 \\
\hline
\end{tabular}




\section{VISITE/ASSISTIBILI}

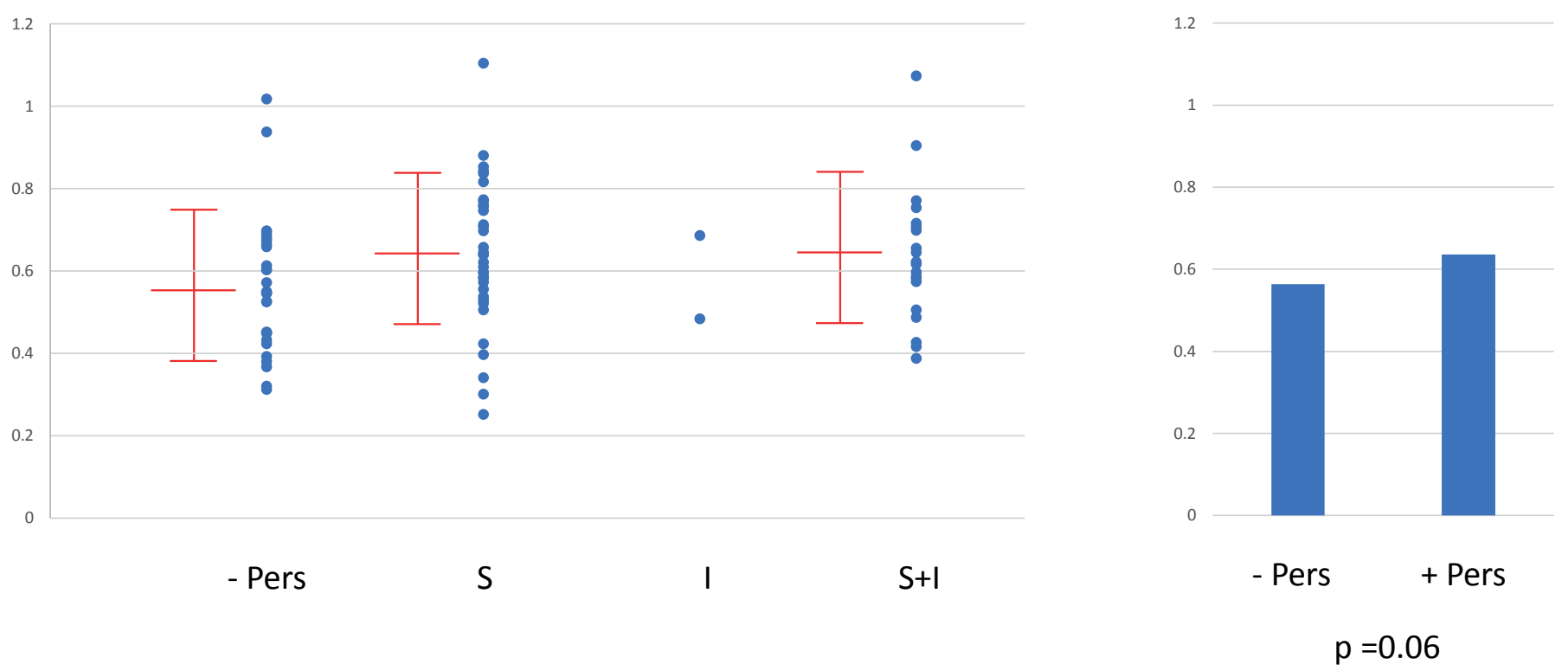

Figura 1. Indicatore visite/assistibili. Nel grafico di sinistra vengono riportate le distribuzioni delle osservazioni in riferimento alla presenza di personale. L'indicazione schematica per le medie e le deviazioni standard relativa a ogni gruppo è riportata a sinistra dei singoli "strip-plot". L'istogramma a destra riporta i valori delle medie del gruppo dei pediatri senza personale e con personale. - Pers: senza personale; S: con personale di segreteria; I: con personale infermieristico; S+l con personale di segreteria e personale infermieristico; + Pers: con personale di segreteria o personale infermieristico o entrambi.

\section{PPIP/VISITE}
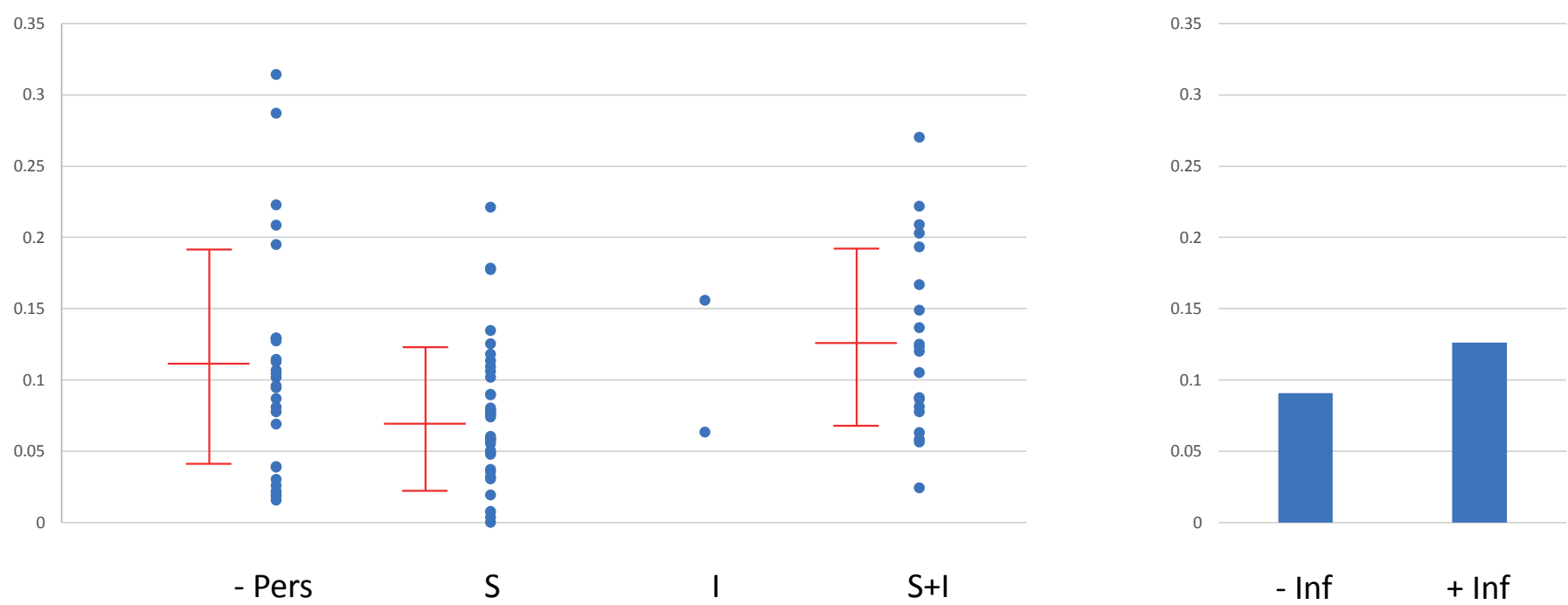

$p=0.03$

Figura 2. Indicatore PPIP/visite. Nel grafico di sinistra vengono riportate le distribuzioni delle osservazioni in riferimento alla presenza di personale. L'indicazione schematica per le medie e le deviazioni standard relativa a ogni gruppo è riportata a sinistra dei singoli "strip-plot". L'istogramma a destra riporta i valori delle medie del gruppo dei pediatri senza personale infermieristico e con personale infermieristico. - Pers: senza personale; S: con personale di segreteria; I: con personale infermieristico; S+I con personale di segreteria e personale infermieristico; - Inf: senza personale infermieristico; + Inf: con personale infermieristico.

stico (Tabella 2, Figura 1). Anche se la differenza fra le medie non raggiunge la significatività statistica, il confronto fra i diversi modelli organizzativi indica che, assumendo un numero di assistibili pari a 1000 , un pediatra con personale ha eseguito in media circa 1,5 visite in più al giorno nel periodo esaminato rispetto ad un pediatra senza collaboratori. L'ACN per la pediatria prevede che l'accesso in ambulatorio venga preferenzialmente regolato attraverso un sistema di prenotazione [3], ormai largamente utilizzato dalle famiglie prevalentemente tramite contatto telefonico. È probabile che il triage telefonico che i medici senza collaboratori conducono in prima persona consenta una maggiore discrezionalità nella programmazione delle visite rispetto a quella consentita a una figura non medica. È tuttavia ipotizzabile, analogamente, che il maggior numero di visite effettuato dai pediatri con personale sia bilanciato da un minor tempo impiegato da questi ultimi nell'organizzazione telefonica dell'accesso all'ambulatorio. L'impatto positivo del lavoro del personale di segreteria e infermieristico sia sull'attività del medico che sulla 

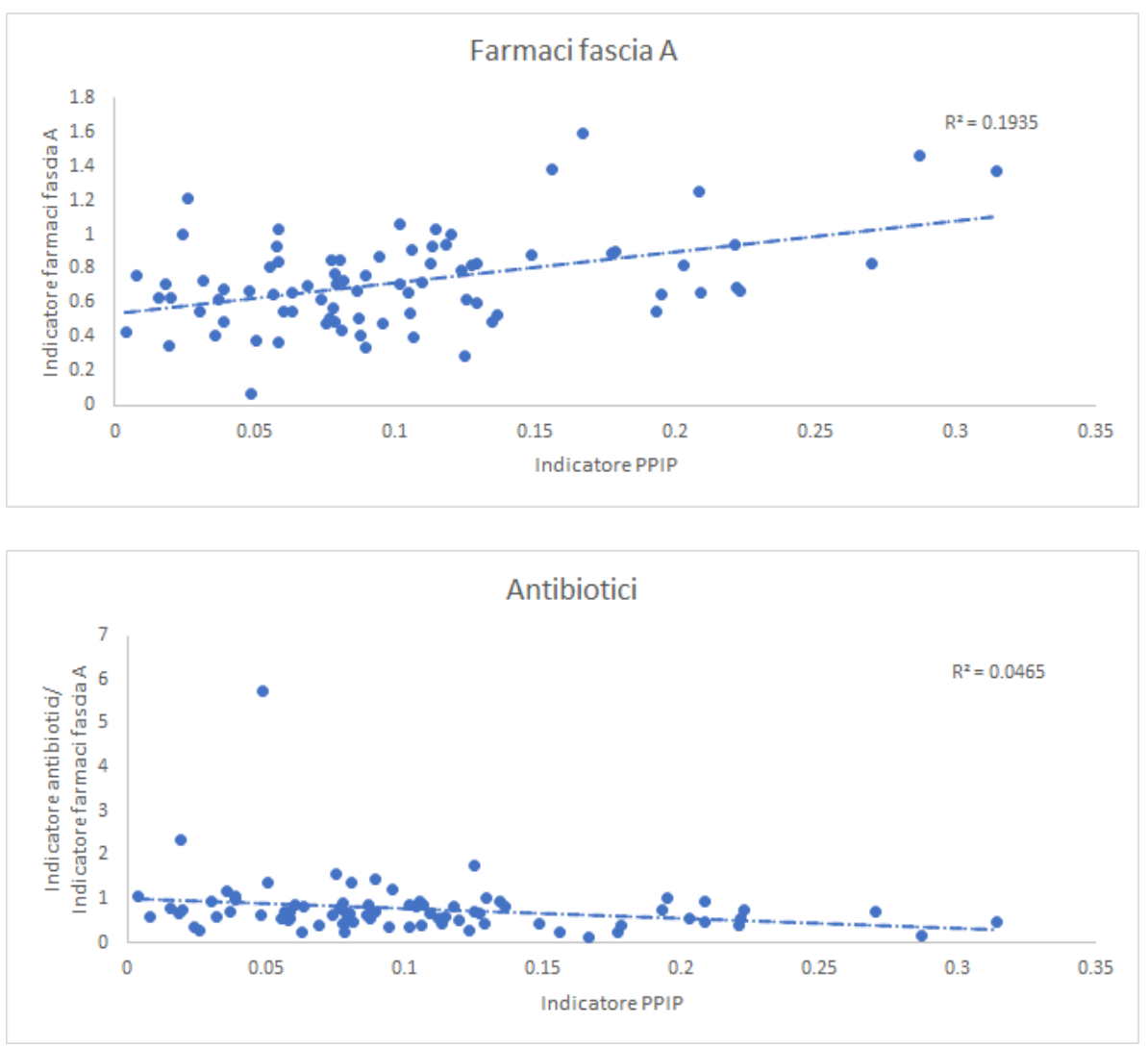

Figura 3. Distribuzione degli indicatori di prescrizione dei farmaci in fascia A e degli antibiotici in relazione all'indicatore delle PPIP. Indicatore farmaci fascia A: numero di confezioni prescritte di farmaci fascia A/visite. Indicatore PPIP: numero di PPIP/visite. Indicatore antibiotici: numero di confezioni prescritte di antibiotici/numero di confezioni prescritte di farmaci in fascia A. La linea tratteggiata indica la retta di regressione lineare. Per ogni grafico, in alto a destra viene riportato valore di $\mathrm{R}^{2}$.

qualità percepita dagli utenti e la necessità di ampliare l'opera di figure affiancanti il medico delle cure territoriali sono state sottolineate da alcuni studi $[10,11]$. L'implementazione di percorsi condivisi di triage telefonico per il personale potrebbe condurre a una maggiore appropriatezza nella selezione di pazienti da sottoporre a osservazione ambulatoriale [12].

La nostra analisi indica che la presenza di personale infermieristico si associa in maniera significativa a un più frequente ricorso alla diagnostica di self-help (Tabella 2, Figura 2). Come già sottolineato da altri studi [5,6], l'utilizzo del self-help, oltre che a una maggiore accuratezza diagnostica e prescrittiva, si associa a un minor numero di accessi inappropriati al pronto soccorso e a un contenimento della spesa farmaceutica. In linea con queste osservazioni, l'analisi di regressione riportata nella Figura piego delle PPIP rispetto agli altri strumenti analizzati (visite, $\mathrm{BdS}$, prescrizione di farmaci). Ci sembra opportuno sottolineare come, mentre sono a disposizione dei PLS linee guida per la prescrizione dei farmaci e chiare indicazioni da parte dell'ACN per l'epoca di esecuzione dei BdS [3], vi sono pochi documenti ufficiali relativi all'utilizzo del self-help nell'ambulatorio del pediatra [13]. Sebbene, come di sopra indicato, un maggiore ricorso alle PPIP possa essere in parte ricondotto alla presenza in alcuni ambulatori di personale infermieristico, quest'ultimo elemento non è sufficiente da solo a giustificare l'eterogeneità del comportamento riscontrato fra i PLS partecipanti. L'osservazione che il ricorso allo strumento PPIP sia disomogeneo anche fra PLS operanti all'interno di una stessa area geografica (dati non riportati) rende meno probabile che tale disomogeneità sia da ricondurre a una diversa occorrenza della patologia febbrile in differenti aree territoriali durante il periodo in esame. Nel nostro studio, a fronte di 8 PLS che hanno dichiarato di aver effettuato più di 100 PPIP, altri 7 PLS hanno riportato di aver eseguito meno di 10 PPIP nell'intervallo di tempo considerato (pur da ritenere un periodo di patologia acuta), e tra questi ultimi 1 PLS ha dichiarato di non averne eseguita nessuna. Una più precisa indicazione circa l'utilizzo di questo importante strumento di lavoro da parte di linee guida condivise e la partecipazione a percorsi formativi ad hoc non potranno che ridurre l'eterogeneità di impiego riscontrata in questo studio e migliorare la qualità della prestazione della visita ambulatoriale fornita dai pediatri di famiglia ai pazienti con patologia acuta.

In conclusione, l'analisi dei dati forniti da questo studio suggerisce l'implementazione di protocolli e linee guida specifici per la pediatria territoriale riguardanti l'uso di comuni strumenti di lavoro, che includano il ruolo sempre più importante affidato al personale paramedico e amministrativo nella gestione ambulatoriale.

damagio@iol.it

I ringraziamenti e la bibliografia sono consultabili online. 
Bibliografia

1. Montanari Vergallo G, Zaami S. Guidelines and best practices: remarks on the Gelli-Bianco law. Clin Ter. 2018;169:e82-e85

2. Van Esso D, del Torso S, Hadjipanayis A et al. Paediatric primary care in Europe: variation between countries. Arch Dis Child. 2010;95:791-5

3. ACN per la pediatria di famiglia 15/12/2005 ai sensi dell'art. 8 del d.lgs. n. 502 del 1992 e successive modificazioni e integrazioni. http://www.sisac.info/

4. Cohen JF, Bertille N, Cohen R, et al. Rapid antigen detection test for group A streptococcus in children with pharyngitis. Cochrane Database Syst Rev 2016;7:CD010502.

5. Nova A, Narducci M, Zanetto F et al. La prescrizione antibiotica sistemica nella pratica del pediatra di famiglia. Medico e Bambino 2008;15:106-11.

6. Mauri L, Narducci M, Nova, A, et al. Infezioni respiratorie, self help e prescrizione antibiotica nella pratica ambulatoriale. Medico e Bambino 2010;29:569-74

7. Clavenna A, Sequi M, Bortolotti A et al. Farmaci essenziali e attitudini prescrittive dei pediatri. Medico e Bambino 2010;29:565-9

8. Bonicelli I, Carrozzo R, Bacchini M et al. Sars-CoV-2 e pediatria territoriale: Armageddon o stimolo per riorganizzare il nostro modo di lavorare? Medico e Bambino 2020;39:174-9

9. Marinello R, Picca M, Freri D, et al. La pediatria di famiglia nell'area milanese: un'indagine di customer satisfaction. Medico e Bambino pagine elettroniche 2011;14 https://www.medicoebambino.com/?id=RIC1110_10.html

10. Litchfield I, Gale N, Burrows M et al. The future role of receptionists in primary care. Br J Gen Pract. 2017;67:523-524

11. Cawley JF. Physician assistants and their role in primary care. Virtual Mentor. 2012;14:411-4

12. Greco L. Manuale di qualità per la pediatria di famiglia. Pacini Editore, 2004

13. Faringotonsillite in età pediatrica. Guida Rapida 2015. assr.regione.emilia-romagna.it 